\title{
TOPOLOGICAL STRUCTURE OF SOLUTION SETS OF DIFFERENTIAL INCLUSIONS: THE CONSTRAINED CASE
}

\author{
WOJCIECH KRYSZEWSKI
}

Received 11 March 2002

We survey and announce some current results on the existence, the viability, and the topological structure of the viable solutions of differential equations and inclusion in Banach spaces under set constraints. Some new results concerning semilinear differential inclusions with state variables constrained to the so-called regular and strictly regular sets, together with their applications, are presented and discussed.

\section{Introduction}

It is our purpose to study solutions of the Cauchy problem for a semilinear differential inclusion

$$
\begin{gathered}
u^{\prime}(t) \in A u(t)+\varphi(t, u(t)), \quad t \in J, u \in D, \\
u\left(t_{0}\right)=x_{0} \in D,
\end{gathered}
$$

where $\varphi: J \times D \multimap E$ is an upper-Carathéodory set-valued map, $J$ is an interval (i.e., a connected subset) of the real axis $\mathbb{R}, t_{0} \in J, D$ is a closed subset of a Banach space $E$, and $A$ is the infinitesimal generator of a $C_{0}$-semigroup $\{U(t)\}_{t \geq 0}$ of bounded linear operators on $E\left(A \equiv 0\right.$ and/or $E=\mathbb{R}^{N}$ is not excluded). Problem (1.1) with $D=E$ was studied by many authors-see the monographs $[43,44,46,51]$ and the rich bibliography therein-and diversity of results has been obtained. Here, we address the question of the topological characterization of the set of all solutions (understood in an appropriate sense) of (1.1) under rather weak assumptions concerning the geometry of $D$ (satisfied, e.g., if $D$ is convex) and some natural boundary conditions. If $\varphi$ is defined on $J \times E$, then our problem is intimately related to the viability or the invariance properties of $D$ (see $[5,6]$ ) with respect to $\varphi$, that is, the existence of a solution $u: J \rightarrow E$ 
of (1.1) such that $u(t) \in D$ on $J$; however, we also determine the topological structure of the set of such viable solutions. Problems of this kind seem to be of importance not only from the viewpoint of the academic interest. An appropriate characterization of the set of viable solutions and the use of topological methods involving the fixed-point index theory helps to establish the existence of the constrained periodic dynamics and stationary points (equilibria) of the system governed by the above differential inclusion.

We first state the standing hypotheses $\left(\mathrm{H}_{i}\right), i=1, \ldots, 5$, and recall some preliminary terminology. Throughout the paper, $E$ stands for a separable Banach space.

$\left(\mathrm{H}_{1}\right) \varphi: J \times D \multimap E$ is a set-valued upper-Carathéodory map; that is, for each $t \in J, x \in D$, the value $\varphi(t, x)$ is a nonempty, compact, and convex subset $E$; the map $\varphi(t, \cdot): D \multimap E$ is upper semicontinuous and $\varphi(\cdot, x): J \multimap E$ is measurable. (See, e.g., [31] or [8] for the concepts of continuity and measurability of setvalued maps; we only remark that, under separability, assumption measurability coincides with strong (Bochner) measurability.) Upper-Carathéodory maps enjoy the weak superpositional measurability property: for a continuous $u: J \rightarrow D$, $\varphi(\cdot, u(\cdot))$ possesses a measurable selection $w(\cdot)$. Hence, from the viewpoint of the solvability of (1.1), the regularity requirements seem to be rather minimal. Simple examples show that we cannot dispense with the convexity assumption.

$\left(\mathrm{H}_{2}\right) \varphi$ has linear growth; that is, there is a $c \in L_{\mathrm{loc}}^{1}(J, \mathbb{R}) \operatorname{such}_{\text {that }} \sup _{z \in F(t, x)}\|z\|$ $\leq c(t)(1+\|x\|)$ on $J \times D$. Therefore, $\varphi$ induces the set-valued (Nemytskij) operator $N_{\varphi}$ defined on the set $C(J, D)$ of continuous functions $J \rightarrow D$ into the space $L_{\text {loc }}^{1}(J, E)$ of (locally) Bochner integrable functions $J \rightarrow E$ given by

$$
N_{\varphi}(u):=\left\{w \in L_{\text {loc }}^{1}(J, E) \mid w(t) \in \varphi(t, u(t)) \text { a.e. on } J\right\}
$$

$\left(\mathrm{H}_{3}\right) \varphi$ transforms precompact subsets of $J \times D$ into compact ones.

This assumption is automatically satisfied if $\varphi$ is (jointly) upper semicontinuous or if $\operatorname{dim} E<\infty$ and $c \equiv$ const and seems to be a minimal compactness condition required in an infinite dimensional setting and in the presence of constraints.

$\left(\mathrm{H}_{4}\right)$ A closed densely defined linear operator $A: E \supset D(A) \rightarrow E$ is the infinitesimal generator of a $C_{0}$-semigroup $U=\{U(t)\}_{t \geq 0}$ such that $\|U(t)\| \leq \exp (\omega t)$ where $\omega \in \mathbb{R}$ for $t \geq 0$.

It is clear (using an appropriate renorming procedure) that this does not restrict generality (for details, cf. [50, Chapter VII] and [53, 58]).

$\left(\mathrm{H}_{5}\right) D$ is a closed subset $E$, invariant with respect to $U$, that is, $U(t) D \subset D$, $t \geq 0$.

This condition may be stated in terms of $A$ only (see [50, Proposition VII.5.3, Remark VII.5.2] and [51]) holds if and only if $\liminf _{t \rightarrow 0^{+}} d_{D}(U(t) x) / t=0$ for all 
$x \in D$, where $d_{D}$ is the distance function

$$
d_{D}(x):=\inf _{y \in D}\|x-y\|, \quad x \in E .
$$

Given $x_{0} \in E, t_{0} \in J$, and $f \in L_{\mathrm{loc}}^{1}(J, E)$, the function

$$
M\left(x_{0}, t_{0} ; f\right)(t)=U\left(t-t_{0}\right) x_{0}+\int_{t_{0}}^{t} U(t-s) f(s) d s, \quad t \in J
$$

is, by definition, the mild solution of the initial value problem

$$
u^{\prime}(t)=A u(t)+f(t), \quad u\left(t_{0}\right)=x_{0} .
$$

Note that even the continuity of $f$ does not imply that (1.5) has a strong solution, that is, an almost everywhere (a.e.) differentiable function $u: J \rightarrow E$ such that $u^{\prime} \in L_{\mathrm{loc}}^{1}(J, E), u\left(t_{0}\right)=x_{0}$, and $u^{\prime}(t)=A u(t)+f(t)$ a.e. on $J$; however, if $u$ is uniformly continuous (i.e., $\|I-U(t)\| \rightarrow 0$ when $t \rightarrow 0^{+}$), or the function $v: t \mapsto$ $\int_{t_{0}}^{t} U(t-s) f(s) d s$ is differentiable a.e. with $v^{\prime} \in L_{\mathrm{loc}}^{1}$ and $x_{0} \in D(A)$, then the mild solution is a (unique) strong solution (see [53]).

A continuous function $u: J \rightarrow D$ is a mild solution to (1.1) if there is $w \in$ $N_{\varphi}(u)$ such that $u=M\left(x_{0}, t_{0} ; w\right)$; hence, the set $S\left(x_{0}, t_{0}\right)$ of all mild solutions of (1.1) coincides with the set of fixed points of the set-valued operator $M\left(x_{0}, t_{0} ; \cdot\right) \circ$ $N_{\varphi}$ defined on $C(J, D)$.

To state the results, we need to recall some other concepts. By the Bouligand and Clarke tangent cones to $D$ at $x \in D$, we understand the cones

$$
\begin{aligned}
& T_{D}(x):=\left\{y \in E: \liminf _{h \rightarrow 0^{+}} \frac{d_{D}(x+h y)}{h}=0\right\}, \\
& C_{D}(x)=\left\{u \in E \mid \lim _{h \rightarrow 0^{+}, y \stackrel{D}{\rightarrow} x} \frac{d_{D}(y+h u)}{h}=0\right\},
\end{aligned}
$$

respectively ( $y \stackrel{D}{\rightarrow} x$ means that $y$ converges to $x$ remaining in $D$ ). Observe that $C_{D}(x)$ is a closed convex cone and $C_{D}(x) \subset T_{D}(x)$. If $D$ is convex, then $T_{D}(x)=$ $C_{D}(x)$ (see [8] for details).

Given a locally Lipschitz continuous function $f: E \rightarrow \mathbb{R}$, by $f^{\circ}(x ; u)$ we denote the Clarke generalized directional derivative of $f$ at $x \in E$ in the direction $u \in E$. The Clarke generalized gradient of $f$ at $x, \partial f(x):=\left\{p \in E^{*} \mid\langle p, u\rangle \leq\right.$ $f^{\circ}(x ; u)$ for all $\left.u \in E\right\}$, is a nonempty $w^{*}$-compact convex subset of $E^{*}$ and $f^{\circ}(x ; u)=\sup _{p \in \partial f(x)}\langle p, u\rangle$. It is clear that $u \in \partial f(x)^{-}$(where $\partial f(x)^{-}:=\{u \in E \mid$ $\langle p, u\rangle \leq 0$ for all $p \in \partial f(x)\}$ is the negative polar cone) if and only if $f^{\circ}(x ; u) \leq$ 0 . In particular, $C_{D}(x)=\partial d_{D}(x)^{-}$for all $x \in D$ (see [21] for details).

Given a bounded $\Omega \subset E$,

$$
\begin{aligned}
& \alpha(\Omega):=\inf \{\varepsilon>0 \mid \Omega \text { admits a finite covering by sets of diameter } \leq \varepsilon\}, \\
& \beta(\Omega):=\inf \{\varepsilon>0 \mid \Omega \text { admits a finite covering by } \varepsilon \text {-balls }\}
\end{aligned}
$$


are the Kuratowski and Hausdorff measures of noncompactness, respectively. These measures are regular, monotone, and nonsingular, that is, for $\gamma=\alpha$ or $\beta$, we have $\gamma(\Omega)=0$ if and only if $\Omega$ is precompact, $\gamma(\Omega) \leq \gamma\left(\Omega^{\prime}\right)$ if $\Omega \subset \Omega^{\prime}$, and $\gamma(\{x\} \cup \Omega)=\gamma(\Omega)$ for each $x \in E$ (see [1] for details).

In the space $C(J, E)$ of continuous functions $J \rightarrow E$, we consider the compactopen topology. Thus, $C(J, E)$ is a Fréchet (locally convex metrizable and complete) space with the metric

$$
\rho(u, v):=\max _{k \geq 1} \frac{2^{-k} p_{k}(u-v)}{1+p_{k}(u-v)}, \quad u, v \in C(J, E),
$$

where $p_{k}(u)=\sup _{t \in J_{k}}\|u(t)\|$ and $\left\{J_{k}\right\}_{k=1}^{\infty}$ is a family of compact intervals such that $J_{k} \subset$ int $J_{k+1}$ and $J=\bigcup_{k=1}^{\infty} J_{k}$. Thus, $C(J, E)$ has the projective topology introduced by restrictions $\left\{\pi_{k}: C(J, E) \rightarrow C\left(J_{k}, E\right) \mid k \geq 1\right\}, \pi_{k}(u)=u \mid J_{k}$ for $u \in$ $C(J, E)$. A set $S \subset C(J, E)$ is compact if and only if $S^{k}:=\pi_{k}(S)$ is compact for each $k \geq 1$. It is also easy to see that $S$ is homeomorphic to the inverse limit $\operatorname{liminv}_{k \rightarrow \infty} S^{k}$ of the inverse system $\left\{S^{k}, \pi_{k l}\right\}$, where $\pi_{k l}$ is the restriction of functions from $S^{k}$ to $J_{l}(l \leq k)$.

\section{Existence}

Among many existence results (see, e.g., $[43,44,51,57]$ or [46] with huge bibliography), the one due to Bothe [16, Theorem 7.2, Corollary 7.1] seems to be the most general.

Theorem 2.1. Assume that

$$
\varphi(t, x) \cap T_{D}(x) \neq \varnothing \quad \text { for a.e. } t \in J \text { and all } x \in D \text {. }
$$

Then, the set-valued map $S: D \times J \multimap C(J, D)$, assigning to $\left(x_{0}, t_{0}\right) \in D \times J$ the set $S\left(x_{0}, t_{0}\right)$ of all mild solutions of (1.1), is upper semicontinuous with nonempty compact values provided one of the following conditions holds:

(i) for any bounded $\Omega \subset D$,

$$
\lim _{h \rightarrow 0^{+}} \beta(\varphi(J(t, h) \times \Omega)) \leq k(t) \beta(\Omega) \quad \text { for each } t \in J,
$$

where $J(t, h):=(t-h, t+h) \cap J$ and $k \in L_{\text {loc }}^{1}(J, \mathbb{R})$; or

(ii) the semigroup $U$ is compact ( $U$ is compact if $U(t)$ is compact for all $t>0$ ).

Remark 2.2. (1) Observe that a compactness assumption (2.2) implies $\left(\mathrm{H}_{3}\right)$. Moreover, if $D=E$, then a weaker condition

$$
\beta(\varphi(\{t\} \times \Omega)) \leq k(t) \beta(\Omega) \quad \text { on } J
$$

for bounded $\Omega \subset D$ (i.e., (2.2) with $J(t, h)=\{t\}$ ) is also sufficient for the assertion of Theorem 2.1 (see [46]). If $D \neq E$, then (2.3), together with $\left(\mathrm{H}_{3}\right)$, is also sufficient provided we know more about $D$-see Theorems 5.4 and 6.6. 
(2) In particular, if $f: J \times D \rightarrow E$ is single valued, for any $x \in D, t \in J, f(\cdot, x)$ is measurable and maps compact subsets of $J$ into compact sets, $f(t, \cdot)$ is locally Lipschitz (uniformly with respect to $t$ from compact subsets of $J$ ) and satisfies (2.1), then (1.1) has a unique mild solution which depends continuously on $\left(x_{0}, t_{0}\right) \in D \times J$. Indeed, under these assumption, $f$ satisfies all hypotheses of Theorem 2.1 locally; hence, a local unique mild solution exits. To establish the result, we apply the usual continuation method.

Observe that, for any $x \in D, y \in E$, and $h>0$,

$$
d_{D}(U(h) x+h y) \leq h\|U(h) y-y\|+d_{D}(U(h)(x+h y))
$$

thus, condition (2.1) implies

$$
F(t, x) \cap T_{D}^{u}(x) \neq \varnothing \quad \text { for a.e. } t \in J \text { and all } x \in D \text {, }
$$

where

$$
T_{D}^{\mathcal{U}}(x):=\left\{y \in E: \liminf _{h \rightarrow 0^{+}} \frac{d_{D}(U(h) x+h y)}{h}=0\right\}
$$

(in general, $T_{D}^{\Upsilon}(x)$ is not a cone). Theorem 2.1 has been proved under assumption (2.5) instead of (2.1). Condition (2.5) is strictly weaker than our (2.1). To see this, consider $E=\mathbb{R}, U(t)=e^{-t}$ for $t \geq 0$, and let $D=[-1,1]$. Then, $D$ is invariant with respect to $U(t)$ (i.e., $U(t) D \subset D)$, but $T_{D}(1)=(-\infty, 0] \subset(-\infty, 1]=$ $T_{D}^{Q \ell}(1)$. Both conditions (2.1) and (2.5) are natural since we have the following proposition.

Proposition 2.3. Condition (2.5) is necessary for the existence of solutions of (1.1). Precisely, if $\varphi$ is upper semicontinuous and, for every $x_{0} \in D$ and $t_{0} \in J$, (1.1) has a mild solution, then (2.5) is satisfied.

Next, in view of the inequality

$$
\left|d_{D}(x+h(A x+y))-d_{D}(U(h) x+h y)\right| \leq\|x+h A x-U(h) x\|
$$

valid for all $x \in D(A)$, we see that (2.5) implies

$$
[A x+F(t, x)] \cap T_{D}(x) \neq \varnothing \quad \text { for every } t \in J, x \in D \cap D(A) .
$$

In case $E=\mathbb{R}^{N}$, (then, $A$ is defined everywhere and bounded) condition (2.8) is sufficient and necessary for the existence. Simple, constructive and based on the technique of the so-called proximal aiming, proof of this fact is given in [14]. 
Moreover, (2.8) may be relaxed (instead of $T_{D}(\cdot)$, we can consider the convex envelope conv $\left.T_{D}(\cdot)\right)$.

Some other tangency conditions leading to the existence in the unconstrained case are considered, for example, in $[19,32]$ or [51].

\section{Solution sets of the semilinear system}

A classical result of Aronszajn [4] states that the solution set for the Cauchy problem in $\mathbb{R}^{N}$ is a compact $R_{\delta}$-set; it is also true for differential inclusions-see for example, [25, 40, 41] (autonomous systems), [26, 38] (nonautonomous), cf. [7, Corollary 5, page 109] and the surveys [33, 38]; asymptotic problems have been studied recently for example in $[2,3]$. (This paper provides an extensive survey on the characterization of the fixed-point set of set-valued maps.)

Recall that a compact metric space $X$ is an $R_{\delta}$-set if there is an ANR (absolute neighborhood retract) $Y$ containing $X$ as a closed subspace such that $X$ is contractible in each of its open neighborhoods (i.e., given an open neighborhood $V$ of $X$ in $Y$, there is a continuous map $h: X \times[0,1] \rightarrow V$ with $h(x, 0)=x$ and $h(x, 1)=x_{0} \in V$, for all $\left.x \in X\right)$. ( $R_{\delta}$-sets are sometimes called cell-like sets.) The $R_{\delta}$-property is a homotopy invariant: if compacta $X_{1}$ and $X_{2}$ are homotopy equivalent and $X_{1} \in R_{\delta}$, then so is $X_{2}$. Similarly, given an ANR $Z$ containing $X$ as a closed subspace, if $X \in R_{\delta}$, then $X$ is contractible in each of its neighborhoods in $Z$. It is clear that if $X$ is a subset of a metric space $T$ and there is a decreasing family $\left\{X_{n}\right\}_{n \geq 1}$ of closed contractible sets such that $\gamma\left(X_{n}\right) \rightarrow 0$, where $\gamma$ is a regular, monotone and nonsingular measure of noncompactness on $T$, then $X \in R_{\delta}$. The celebrated result of Hyman [45] states that if $X \in R_{\delta}$, then there is a decreasing sequence of contractible compacta $\left\{X_{n}\right\}$ containing $X$ as a closed subspace such that $X=\bigcap_{n \geq 1} X_{n}$. $R_{\delta}$-sets are connected, have trivial shape, and are acyclic with respect to any continuous (co)homology theory, that is, they have the same (co)homology as a one-point space. Recall (see [35]) that if $X$ is homeomorphic to the inverse limit $\operatorname{liminv}_{n \geq 1} X_{n}$ of the countable inverse system $\left\{X_{n} ; \pi_{n m}: X_{m} \rightarrow X_{n}, n \leq m\right\}$ and $X_{n} \in R_{\delta}$ for all $n$, then $X \in R_{\delta}$. In particular, $S \subset C(J, E)$ is $R_{\delta}$ if and only if, for all $n \geq 1$, the set $S^{k}=\pi_{k}(S)$ of restrictions of functions from $S$ to $J_{k}$ is $R_{\delta}$.

We establish the $R_{\delta}$-structure of the set $S\left(x_{0}, t_{0}\right)$ of mild solutions of the constrained semilinear system (1.1). This result is known when $D=E$ (see $[16,46])$; it is also believed that, in fact, the existence implies the $R_{\delta}$-structure (see [20]). See also $[22,43,44]$ for other results and many bibliographical comments. The constrained case seems to be more involved. The result holds if $D$ is invariant with respect to $U$, convex, and int $D \neq \varnothing$, or if $D$ is convex proximinal (i.e., each $x \in E$ admits a nearest point in $D$ ) and $U$ is nonexpansive (see $[11,17,43,44]$ ) and $\varphi$ obeys the tangency condition (2.1). For the finite-dimensional situation, see $[13,29,30,31,42]$.

To see that even in the convex case the situation is more complicated, we consider the following example. 
Example 3.1 (see [31]). Let $D=[0, \infty), f(x)=2 \sqrt{|x|} \operatorname{sgn} x$, and $x_{0}=0$. Then, $f(x) \in T_{D}(x)=C_{D}(x)=\mathbb{R}$ on $D$, but $u(t)=-t^{2} \notin D$ is a solution of (1.1) (with $\varphi=f, A \equiv 0$, and $\left.t_{0}=0\right)$.

The example shows that even a stronger tangency condition

$$
\varphi(t, x) \subset T_{D}(x)
$$

(or $\varphi(t, x) \subset C_{D}(x)$ ) on $J \times D$ does not prevent some solutions to leave $D$. Hence, in the case $\varphi$ is defined on $J \times E$, the "unconstrained" results fail to help in the characterization of the set of solutions surviving in $D$ : to get convex-constrained results, we need slightly more involved arguments. In the next section, we provide a result valid for a general convex closed $D$.

The topological structure of $S\left(t_{0}, x_{0}\right)$ changes dramatically in case the set $D$ is not convex. We study some examples (for simplicity, we consider a finitedimensional situation with $D$ (automatically) invariant with respect to the semigroup generated by $A \equiv 0$ ).

Example 3.2 (see [31]). Let $D=\left\{(x, y) \in \mathbb{R}^{2} \mid x \geq 0, y=x^{2}\right\} \cup[0, \infty) \times\{0\}$ and $f(x, y)=(1,2 \sqrt{y})$ on $D$. Then, $f(z) \in T_{D}(z)$ for $z \in D$ and the problem $u^{\prime}=$ $f(u), u(0)=0$ has only two solutions: $u(t)=(t, 0)$ and $u(t)=\left(t, t^{2}\right)$ for $t \geq 0$.

Example 3.3. Let $E=\mathbb{R}^{3}, D:=\left\{x=\left(x_{1}, x_{2}, x_{3}\right) \in E|| x \mid \leq \sqrt{2}\right.$ and $\left.\sqrt{x_{1}^{2}+x_{2}^{2}} \geq x_{3}\right\}$, $S:=\left\{x \in D \mid x_{1}^{2}+x_{2}^{2}=1\right.$ and $\left.x_{3}=1\right\}$, and $Z=\left\{x \in E \mid x_{1}^{2}+x_{2}^{2} \leq 1\right.$ and $\left.x_{3}=1\right\}$. Next, for $x \in D$, let

$$
\varphi(x)= \begin{cases}Z & \text { for } x \in D \backslash S, \\ \operatorname{conv}\left\{Z \cup\left\{\left(-x_{2}, x_{1}, 0\right)\right\}\right\} & \text { for } x \in S .\end{cases}
$$

Clearly, $\varphi: D \multimap E$ is upper semicontinuous and $\varphi(x) \cap T_{D}(x) \neq \varnothing$ on $D$. But it is easy to see that $S\left(x_{0}, t_{0}\right)$ (with $t_{0}=0, x_{0}=0$ ) is homeomorphic to the unit sphere $S^{1}:=\left\{x \in \mathbb{R}^{2} \mid\|x\|=1\right\}$; hence, it is not an $R_{\delta}$-set. Notice that, for all $x \in D$, $x \neq 0$, the Bouligand and the Clarke tangent cones $T_{D}(x)$ and $C_{D}(x)$ coincide; however, $T_{D}(0) \neq C_{D}(0)$ and $F(0) \cap C_{D}(0)=\varnothing$.

In the above example, if we consider $\varphi$ satisfying (2.1) with the Clarke cone replacing the Bouligand one, then the situation becomes clear. However, it is not true that such a procedure would be the general remedy.

Example 3.4 (see [14]). Let $D=S_{1} \cup S_{-1}$, where $S_{i}=\left\{x=\left(x_{1}, x_{2}\right) \in \mathbb{R}^{2} \mid\left(x_{1}-i\right)^{2}\right.$ $\left.+x_{2}^{2}=1\right\}$, and, for $x \in D$, let

$$
\varphi(x)= \begin{cases}\left(x_{2}, 1-x_{1}\right) & \text { for } x \in S_{1} \\ \left(-x_{2}, 1+x_{1}\right) & \text { for } x \in S_{-1}\end{cases}
$$


Then, $\varphi(x) \in T_{D}(x)=C_{D}(x)$ on $D$, but $S\left(x_{0}, t_{0}\right)$ (with $t_{0}=0$ and $x_{0}=0$ ) is not connected.

It seems, therefore, that in order to state the correct tangency condition which implies the expected topological structure of solution sets to (1.1), we should replace in (2.1) the Bouligand cones by the Clarke ones, that is, assume that

$$
\forall(t, x) \in J \times D, \quad F(t, x) \cap C_{D}(x) \neq \varnothing
$$

and take care of the geometry of the involved set $D$. The first attempt in this direction (for $E=\mathbb{R}^{N}$ and $A \equiv 0$ ) was done by Plaskacz [54], where he studies the class $\rho$ of sets (called proximate retracts in [37]) and assumes (3.1); Plaskacz's result was extended to the Hilbert space context (see [39]). Up to now, the most general results for the finite dimensional case were given in [13]. Below, we will generalize them to the present infinite dimensional situation.

\section{Convex case}

The general strategy to obtain the $R_{\delta}$-structure of solution sets is to approximate $\varphi$ in an appropriate way by a sequence of auxiliary set-valued maps $\left\{\varphi_{n}\right\}$ possessing locally Lipschitz selections and, then, to show that $S\left(x_{0}, t_{0}\right)$ is an intersection of solution sets corresponding to $\varphi_{n}$. In the constrained case, the main difficulty is to assure that maps $\varphi_{n}$ and their locally Lipschitz selections obey the necessary tangency condition implying existence.

We first deal with the general convex case. We state the result with a sketch of the proof in order to show how the above described procedure works.

For a convex closed subset $X$ of a normed space $Y$ and $x \in X$,

$$
C_{X}(x)=T_{X}(x)=\overline{S_{X}(x)}
$$

where

$$
S_{X}(x):=\bigcup_{h>0} \frac{X-x}{h} .
$$

Our improvement of the mentioned results on the structure of solutions living in convex sets (cf. $[11,18]$ ) is based upon the following lemma.

Lemma 4.1. If $\Phi: X \multimap Y$ is upper semicontinuous with closed convex values and, for each $x \in X$,

$$
\Phi(x) \cap T_{X}(x) \neq \varnothing,
$$

then, for any $\varepsilon>0$, there is a locally Lipschitz map $F: X \rightarrow Y$ such that

$$
\forall x \in X, \quad F(x) \in \Phi\left(B_{X}(x, \varepsilon)\right)+B_{Y}(0, \varepsilon)
$$


(where $B_{X}(x, r):=\{y \in X \mid d(x, y)<r\}$ is the ball in $X$; usually the subindex $X$ is suppressed from the notation) and

$$
\forall x \in X, \quad F(x) \in T_{X}(x) .
$$

Remark 4.2. If $Y$ is complete, then the existence of a continuous (single-valued) map $F: X \rightarrow Y$ satisfying conditions (4.4) and (4.5) follows from a general result from [14]. Here, we need no completeness and improve this result obtaining a locally Lipschitzian $\varepsilon$-selection.

Proof. Take $\varepsilon>0$ and $x \in X$. There is $v(x) \in Y$ such that

$$
v(x) \in \Phi(x)+B_{Y}\left(0, \frac{\varepsilon}{4}\right) \cap S_{X}(x)
$$

in view of (4.3) and (4.1). Hence, by (4.2), there is $\alpha(x)>0$ such that

$$
x+\alpha(x) v(x) \in X
$$

By upper semicontinuity, choose $\gamma(x), 0<\gamma(t, x)<\varepsilon / 4$ such that

$$
\Phi\left(B_{X}(x, 2 \gamma(x))\right) \subset \Phi(x)+B_{Y}\left(0, \frac{\varepsilon}{2}\right)
$$

and $\delta(x), 0<\delta(x)<\min \{\gamma(x), \gamma(x) / \alpha(x)\}$.

Let $\left\{\lambda_{s}: X \rightarrow[0,1]\right\}_{s \in S}$ be a locally finite locally Lipschitz partition of unity refining the open cover $\left\{B_{X}(x, \delta(x) \alpha(x))\right\}_{x \in X}$. For any $s \in S$, there is $x_{s} \in X$ such that supp $\lambda_{s} \subset B_{X}\left(x_{s}, \delta_{s} \alpha_{s}\right)$ where we have put $\delta_{s}:=\delta\left(x_{s}\right)$ and $\alpha_{s}:=\alpha\left(x_{s}\right)$. Additionally, we set $v_{s}:=v\left(x_{s}\right)$ and $\gamma_{s}:=\gamma\left(x_{s}\right)$.

For any $s \in S$, consider a map $F_{s}: X \rightarrow Y$ given by

$$
F_{s}(x):=\frac{1}{\alpha_{s}}\left(x_{s}-x\right)+v_{s}, \quad x \in X .
$$

For $s \in S, x \in X$,

$$
x+\alpha_{s} F_{s}(x)=x_{s}+\alpha_{s} v_{s} \in X
$$

in view of (4.7); hence,

$$
F_{s}(x) \in S_{X}(x) \subset T_{X}(x)
$$

Clearly, $F_{s}, s \in S$, is Lipschitz continuous (with the Lipschitz constant $\alpha_{s}^{-1}$ ).

A map $F: X \rightarrow Y$ defined by the formula

$$
F(x):=\sum_{s \in S} \lambda_{s}(x) F_{s}(x), \quad x \in X
$$

satisfies the requirements of Lemma 4.1. 
Remark 4.3. In the course of the proof, we have not used the lower semicontinuity of $T_{X}(\cdot)$. Instead, the following astonishingly simple observation (already employed in a different situation in [24]) has been used.

If $X$ is a convex closed subset of a normed space $Y$, then, for every $x_{0} \in$ $X, v_{0} \in S_{X}\left(x_{0}\right)$ and $\alpha_{0}>0$ such that $x_{0}+\alpha_{0} v_{0} \in X$, an affine mapping $g(x)=$ $\left(1 / \alpha_{0}\right)\left(x_{0}-x\right)+v_{0}, x \in X$, provides a selection of $S_{X}(x)$. This proves the lower semicontinuity of both $S_{X}(\cdot)$ and $T_{X}(\cdot)$.

Theorem 4.4. If $D$ is convex and (2.1) holds, then $S\left(x_{0}, t_{0}\right)$ is an $R_{\delta}$-set in $C(J, E)$ provided that (2.2) is satisfied or $U$ is compact.

Proof. To illustrate the setting, we consider an upper-semicontinuous $\varphi$. Take $\left(x_{0}, t_{0}\right) \in D \times J$ and choose a family $\left\{J_{k}=\left[a_{k}, b_{k}\right]\right\}_{k=1}^{\infty}$ of compact subintervals in $J$ such that $J_{k} \subset \operatorname{int} J_{k+1}, \bigcup J_{k}=J$, and $t_{0} \in J_{k}$ for all $k \geq 1$. Fix $k \geq 1$ and let $\Phi: J_{k} \times D \multimap Y:=\mathbb{R} \times E, \Phi(t, x)=\{0\} \times \varphi(t, x)$ (in $Y$, we consider the norm $\|(t, x)\|=\max \{|t|,\|x\|\})$. By Lemma 4.1, there is a locally Lipschitz $F_{n}: J_{k} \times D \rightarrow$ $Y$ such that $F_{n}(t, x) \in T_{J_{k} \times D}(t, x)$ and $F_{n}(t, x) \in \Phi\left(B\left((t, x), n^{-1}\right)\right)+B_{Y}\left(0, n^{-1}\right)$ on $J_{k} \times D(n \geq 1)$. Define $f_{n}:=p \circ F_{n}$ where $p: Y \rightarrow E$ is the projection $p(t, x)=x$. Then, $f_{n}(t, x) \in \varphi_{n}(t, x):=\operatorname{conv} \varphi\left(B\left((t, x), n^{-1}\right)\right)+B\left(0, n^{-1}\right)$ and $f_{n}(t, x) \in T_{D}(x)$ on $J_{k} \times D$. Clearly, for $n \geq 1, \varphi(t, x) \subset \varphi_{n}(t, x) \subset \varphi_{n+1}(t, x)$ on $J_{k} \times D$; thus,

$$
\varnothing \neq S_{n}^{k}\left(x_{0}, t_{0}\right) \supset S_{n+1}^{k}, \quad S^{k}\left(x_{0}, t_{0}\right) \subset \bigcap_{n \geq 1} S_{n}^{k}\left(x_{0}, t_{0}\right)
$$

where $S^{k}\left(x_{0}, t_{0}\right)$ (resp., $\left.S_{n}^{k}\left(x_{0}, t_{0}\right)\right)$ stands for the set of all mild solutions on $J_{k}$ of (1.1) (resp., of (1.1) with $\varphi$ replaced by $\varphi_{n}$ ).

Let $u_{n} \in S_{n}^{k}\left(x_{0}, t_{0}\right)$ for all $n \geq 1$. We then show that there exists a subsequence $\left(u_{n_{m}}\right)_{m \geq 1}$ such that $u_{n_{m}} \rightarrow u_{0} \in S^{k}\left(x_{0}, t_{0}\right)$ in $C\left(J_{k}, E\right)$ as $m \rightarrow \infty$. It follows that

$$
\begin{gathered}
S^{k}\left(x_{0}, t_{0}\right)=\bigcap_{n=1}^{\infty} \overline{S_{n}^{k}\left(x_{0}, t_{0}\right)}, \\
\beta_{0}\left(S_{n}^{k}\left(x_{0}, t_{0}\right)\right) \longrightarrow 0 \text { as } n \longrightarrow \infty,
\end{gathered}
$$

where $\beta_{0}$ stands for the Hausdorff measure of noncompactness in $C\left(J_{k}, E\right)$.

For each $n \geq 1, z \in J_{k}$, and $y \in D$, the problem

$$
v^{\prime} \in A v+f_{n}(t, v), \quad v(z)=y
$$

admits a unique solution $v_{n}(\cdot ; z, y):\left[a_{k}, b_{k}\right] \rightarrow D$, which depends continuously on $(z, y)$ (see Remark 2.2). Define a homotopy $h:[0,1] \times \overline{S_{n}^{k}\left(x_{0}, t_{0}\right)} \rightarrow C(J, E)$ by 
the formula

$$
\begin{array}{r}
h(\lambda, u)(s) \\
::=\left\{\begin{array}{lc}
u(s) & \text { if } s \in\left[a_{k}+\lambda\left(t_{0}-a_{k}\right),\right. \\
\left.b_{k}+\lambda\left(t_{0}-b_{k}\right)\right], \\
v_{n}\left(s ; a_{k}+\lambda\left(t_{0}-a_{k}\right), u\left(a_{k}+\lambda\left(t_{0}-a_{k}\right)\right)\right. & \text { if } s \in\left[a_{k}, a_{k}+\lambda\left(t_{0}-a_{k}\right)\right], \\
v_{n}\left(s ; b_{k}+\lambda\left(t_{0}-b_{k}\right), u\left(b_{k}+\lambda\left(t_{0}-b_{k}\right)\right)\right. & \text { if } s \in\left[b_{k}+\lambda\left(t_{0}-b_{k}\right), b_{k}\right]
\end{array}\right.
\end{array}
$$

for $u \in \overline{S_{n}^{k}\left(x_{0}, t_{0}\right)}$ and $\lambda \in[0,1]$. It is easy to see that $h$ is continuous. Moreover, we have $h\left([0,1] \times S_{n}^{k}\left(x_{0}, t_{0}\right)\right) \subset S_{n}^{k}\left(x_{0}, t_{0}\right)$. From the continuity of $h$, we infer that $h\left([0,1] \times \overline{S_{n}^{k}\left(x_{0}, t_{0}\right)}\right) \subset \overline{S_{n}^{k}\left(x_{0}\right)}$. Finally, observe that $h(1, u)=v_{n}\left(\cdot ; t_{0}, x_{0}\right)$ and $h(0, u)=u$ for every $u \in \overline{S_{n}^{k}\left(x_{0}, t_{0}\right)}$, that is, $\overline{S_{n}^{k}\left(x_{0}\right)}$ is contractible. Hence, $S^{k}\left(x_{0}, t_{0}\right) \in R_{\delta}$ and so is $S\left(x_{0}, t_{0}\right)$.

\section{Epi-Lipschitz case}

An important role in optimization is played by the so-called epi-Lipschitz sets. This notion (in the finite-dimensional context) has been introduced by Rockafellar [56]. The corresponding notion for subsets of a Banach space has been studied in [14].

Definition 5.1. A closed set $D \subset E$ is epi-Lipschitz if, for all $x_{0} \in D$, there are a neighborhood $U$ of $x_{0}$ (in $E$ ), a Banach space $Z$, a topological isomorphism $L: Z \times \mathbb{R} \rightarrow E$ with $L\left(z_{0}, \lambda_{0}\right)=x_{0}$, and a locally Lipschitz function $g: Z \rightarrow \mathbb{R}$ such that

$$
D \cap U=U \cap L(\text { Epig })
$$

where Epi $g:=\{(z, \lambda) \mid g(z) \leq \lambda\}$ is the epigraph of $g$.

Proposition 5.2 (see $[21,23,56]$ ). If $D \subset E=\mathbb{R}^{n}$ is closed, then the following conditions are equivalent:

(i) $D$ is epi-Lipschitz;

(ii) for any $x \in D$, int $C_{D}(x) \neq \varnothing$ (or, equivalently, the Clarke normal cone $N_{D}(x):=C_{D}(x)^{-}$is pointed, that is, $\left.N_{D}(x) \cap\left(-N_{D}(x)\right)=\{0\}\right)$;

(iii) for any $x \in \partial D, C_{D}(x)=\partial \Delta_{D}(x)^{-}$, where, for $y \in \mathbb{R}, \Delta_{D}(y):=d_{D}(y)-$ $d_{E \backslash \text { int } D}(y)$ and $0 \notin \partial \Delta_{D}(x)$.

Implication (i) $\Rightarrow$ (ii) is obvious. Implication (iii) $\Rightarrow$ (ii) follows since if $0 \notin$ $\partial \Delta_{D}(x)$ for $x \in \partial D$, then $\partial \Delta_{D}(x)^{-} \subset C_{D}(x)$ (see [21, Theorem 2.4.7]); hence, $N_{D}(x) \subset\left(\partial \Delta(x)^{-}\right)^{-}=\bigcup_{\lambda \geq 0} \lambda \partial \Delta_{D}(x)$ and $N_{D}(x)$ is pointed. Both these facts hold if $\operatorname{dim} E=\infty$. In order to prove implications (ii) $\Rightarrow$ (i) and (ii) $\Rightarrow$ (iii), we need typically finite-dimensional arguments. The author does not know whether they hold when $\operatorname{dim} E=\infty$. The partial answer is given in the following result. 
Proposition 5.3. If $\operatorname{dim} E \leq \infty$, then (i) $\Rightarrow$ (iii).

THeOrem 5.4. If D is an epi-Lipschitz set and (3.4) is satisfied, then, for each $t_{0} \in J$ and $x_{0} \in D$, the set $S\left(x_{0}, t_{0}\right)$ of all mild solutions of the initial value problem (1.1) is an $R_{\delta}$-set provided the compactness condition (2.3) is satisfied or the semigroup $\boldsymbol{U}$ is compact. One may replace (3.4) by (2.1) in case $\varphi$ is single-valued.

Proof. The last statement is easy. For $x \in \operatorname{int} D, C_{D}(x)=T_{D}(x)=E$. Take $x \in \partial D$ and a sequence $\left(y_{n}\right)$ in $D$ such that $y_{n} \rightarrow x$. Then, for all $t \in J, \varphi\left(t, y_{n}\right) \in T_{D}\left(y_{n}\right)$ and, in view of [ 8 , Theorem 4.1.9],

$$
\varphi(t, x)=\lim _{n \rightarrow \infty} \varphi\left(t, y_{n}\right) \in \operatorname{Liminf}_{y \stackrel{D}{\rightarrow} x} T_{D}(y)=C_{D}(x)
$$

and the first part of the theorem applies.

As concerns the first part, we again construct, for each $n \geq 1$, a map $f_{n}: J \times$ $D \rightarrow E$ such that $f_{n}(\cdot, x)$ is measurable, $f_{n}(t, \cdot)$ is locally Lipschitz (uniformly with respect to $t)$, each point $x \in D$ has a neighborhood $W$ with $f_{n}\left(J_{k} \times W\right)$ lying in a compact subset of $E, f_{n}(t, x) \in C_{D}(x)$, and

$$
f_{n}(t, x) \in \varphi_{n}(t, x):=\operatorname{conv} \varphi\left(\{t\} \times\left[B\left(x, n^{-1}\right) \cap D\right]\right)+B\left(0, n^{-1}\right)
$$

for all $t \in J$ and $x \in D$. The construction recalls that from Lemma 4.1, but it also makes a strong use of the facts that $C_{D}(x)=\partial \Delta_{D}(x)^{-}$and $0 \notin \partial \Delta_{D}(x)$ on $\partial D$. Namely,

$$
f_{n}(t, x)=\sum_{s \in S} \lambda_{s}(x) w_{s}(t)
$$

where $\left\{\lambda_{s}\right\}_{s \in S}$ is an appropriate locally Lipschitz partition of unity and, for $\in S$, $w_{s}: J \rightarrow E$ is a measurable finite-valued function such that $\Delta_{D}^{\circ}\left(y ; w_{s}(t)\right)<0$ for all $t \in J$ and $y \in \operatorname{supp} \lambda_{s}$. Having this, the proof concludes similarly as above.

\section{Regular case}

It is clear that epi-Lipschitz sets have nonempty interiors, and, therefore, neither convex sets nor Plaskacz's proximate retracts are epi-Lipschitz in general. We introduce a class of sets that encompasses epi-Lipschitz or convex sets as well as proximate retracts. Namely, we will deal with the so-called regular domains.

Definition 6.1. We say that a closed set $D \subset E$ is regular if, for any $x \in \partial D$,

$$
\liminf _{y \stackrel{E \backslash D}{\longrightarrow} x}\left\||| \partial d_{D}(y) \mid\right\|>0
$$

where

$$
\left\|\partial d_{D}(x)\right\|\left\|:=\inf _{p \in \partial d_{D}(x)}\right\| p \| .
$$


It is clear that

$$
\left\|\left|\partial d_{D}(x)\right|\right\|=\sup _{\|u\| \leq 1} \inf _{p \in \partial d_{D}(x)}\langle p, u\rangle=-\inf _{\|u\| \leq 1} d_{D}^{\circ}(x ; u) .
$$

Observe that regularity of $D$ means that the distance function $d_{D}$ has no critical points in a neighborhood of $D$ intersected with the complement of $D$. If there is a neighborhood $U$ of $D$ such that $\inf _{y \in U \backslash D}\|\| \partial d_{D}(y) \mid \|>0$, then $D$ is regular; if $D$ is regular compact (or $\partial D$ is compact), then such a neighborhood exists. It appears that regular sets are well designed to study solutions of (1.1) in case of a nonexpansive semigroup $\mathcal{~}$. In order to study a general situation we will also deal with the so-called strictly regular sets.

Definition 6.2. We say that a closed set $D \subset E$ is strictly regular if there is an $r>0$ such that

$$
\inf _{y \in B(D, r) \backslash D}\left\|\left|\partial d_{D}(y)\right|\right\|>0 .
$$

Clearly, strictly regular sets are regular and compact regular sets are strictly regular.

The class of (strictly) regular sets has been introduced in [24] in a different (and a bit more general) setting and studied in the context of equilibria. This class is rich: for instance, the set $D$ in Example 3.3 is strictly regular and the set $D$ from Example 3.4 is not regular.

Example 6.3. (i) Any convex closed set $D \subset E$ is strictly regular: in fact we easily show that $\left\|\partial d_{D}(y)\right\| \| \geq 1$ for all $y \in E \backslash D$ (see [24]).

(ii) Suppose that a closed $D \subset E$ is proximinal, that is, there is a neighborhood $U$ of $D$ such that, for all $y \in U$, the set $\pi_{D}(y):=\left\{z \in D \mid\|y-z\|=d_{D}(y)\right\} \neq$ $\varnothing$. If, for any $y \in U, \pi_{D}(y) \cap \operatorname{Liminf}_{z \rightarrow y} \pi_{D}(z) \neq \varnothing$ (Liminf denotes the lower limit in the sense of Painlevé-Kuratowski (see, e.g., [8, Definition 1.4.6]), then $D$ is regular. Indeed, take $y \in U \backslash D$, let $\bar{x} \in \pi_{D}(y) \cap \operatorname{Liminf}_{z \rightarrow y} \pi_{D}(z)$, put $u:=\bar{x}-$ $y$, and take sequences $y_{n} \rightarrow y, h_{n} \rightarrow 0^{+}$. There is $\bar{x}_{n} \in \pi_{D}\left(y_{n}\right)$ such that $\bar{x}_{n} \rightarrow \bar{x}$. Hence,

$$
\begin{aligned}
& d_{D}\left(y_{n}+h_{n} u\right)-d_{D}\left(y_{n}\right) \\
& \quad \leq h_{n}\left\|\left(\bar{x}_{n}-y_{n}\right)-u\right\|+d_{D}\left(y_{n}+h_{n}\left(\bar{x}_{n}-y_{n}\right)\right)-d_{D}\left(y_{n}\right) .
\end{aligned}
$$

We easily check that $d_{D}\left(y_{n}+h_{n}\left(\bar{x}_{n}-y_{n}\right)\right)=\left(1-h_{n}\right) d_{D}\left(y_{n}\right)$; therefore, $d_{D}^{\circ}(y ; u) \leq$ $-\|u\|$ and $\left\|\partial d_{D}(y)\right\| \| \geq$. If $U$ is a ball around $D$, then $D$ is strictly regular.

(iii) In particular, all proximate retracts (i.e., proximinal closed sets for which $\pi_{D}(x)$ is a singleton for all $x \in U$ ) are regular, $\pi_{D}$, in this case, is continuous.

(iv) If $D$ is epi-Lipschitz, then it is regular.

(v) A smooth (i.e., $C^{1}$ ) Banach submanifold $M \subset E$ of codimension 1 is regular. 
Now, we will discuss in detail the proper tangency condition which leads to the $R_{\delta}$-characterization of the solution set of (1.1). Since values of $\varphi$ are compact and $C_{D}(x)=\partial d_{D}(x)^{-}(x \in D)$, it is clear that (3.4) is equivalent to

$$
\forall x \in D, \quad \sup _{t \in J} \inf _{z \in \varphi(t, x)} d_{D}^{\circ}(x ; z) \leq 0 .
$$

This, in turn, implies that, for all $x \in D$,

$$
\begin{gathered}
\limsup _{y \rightarrow x}\left[\sup _{t \in J} \inf _{z \in \varphi(t, x)} d_{D}^{\circ}(y ; z)\right] \leq 0, \\
\sup _{\varepsilon>0} \inf \sup _{\eta>0} \inf _{y \in B(x, \eta)} \sigma_{\varphi}\left(y ; x^{\prime}\right) \leq \limsup _{y \rightarrow x} \sigma_{\varphi}(y ; x) \leq 0,
\end{gathered}
$$

for all $x \in D$, where, to simplify the notation, we have set

$$
\sigma_{\varphi}(y ; x):=\sup _{t \in J} \inf _{z \in \varphi(t, x)} d_{D}^{\circ}(y ; z) .
$$

Finally, observe that, in case $D$ is compact, (6.8) holds uniformly with respect to $x \in D$, that is,

$$
\sup _{\varepsilon>0} \inf _{\eta>0} \sup _{x \in D, y \in B(x, \eta)} \inf _{x^{\prime} \in B(x, \varepsilon) \cap D} \sigma_{\varphi}\left(y ; x^{\prime}\right) \leq 0 .
$$

Note that the implication $(3.4) \Rightarrow(6.8)$ always holds, while the implication $(3.4) \Rightarrow(6.10)$ is true if $D$ is compact. Our assumptions on $\varphi$ imply that $(6.10) \Rightarrow$ $(6.8) \Rightarrow(3.4)$.

In order to compensate the lack of compactness as concerns $D$ (which seems to be an intrinsic problem in an infinite-dimensional setting), we consider the following condition.

Definition 6.4. We say that $\varphi$ satisfies the uniform tangency condition on $D$ if (6.10) holds locally uniformly on $D$, that is, any point $p \in D$ has a neighborhood $V$ (in $D)$ such that

$$
\sup _{\varepsilon>0} \inf _{\eta>0} \sup _{x \in V, y \in B(x, \eta)} \inf _{x^{\prime} \in B(x, \varepsilon) \cap D} \sigma_{\varphi}\left(y ; x^{\prime}\right) \leq 0 .
$$

The uniform tangency depends on the geometry of the domain $D$.

Example 6.5. Suppose that (3.4) holds. Condition (6.11) holds in each of the following cases:

(i) the set $D$ is proximinal and satisfies assumptions of Example 6.3(ii);

(ii) $D$ is convex and $E$ is reflexive;

(iii) $D$ is compact (then (6.11) holds with $V=D$, that is, (6.10) holds).

Situation (iii) has been discussed above (the conclusion may be achieved via standard compactness arguments). To show (i), we sustain the notation from 
Example 6.3 and take $p \in D$; there is $r>0$ such that $B(p, 2 r) \subset U$. For $\varepsilon>0$, let $\eta \leq \min \{\varepsilon / 2, r\}$ and take $x \in V:=B(p, r) \cap D$ and $y \in B(x, \eta)$. Then, $\|y-p\| \leq$ $2 r$, that is, $y \in U$. Choose $\bar{x} \in \pi_{D}(y) \cap \operatorname{Liminf}_{z \rightarrow y} \pi_{D}(z)$; clearly, $\|x-\bar{x}\|<\varepsilon$. By (3.4), for each $t \in J$, there is $z_{t} \in C_{D}(\bar{x}) \cap \varphi(t, \bar{x})$. Take arbitrary sequences $y_{n} \rightarrow$ $y$ and $h_{n} \rightarrow 0^{+}$. There is a sequence $\bar{x}_{n} \in \pi_{D}\left(y_{n}\right)$ such that $\bar{x}_{n} \rightarrow \bar{x}$, and since $z_{t} \in C_{D}(\bar{x})$, there is a sequence $z_{n} \rightarrow z_{t}$ such that $\bar{x}_{n}+h_{n} z_{n} \in D$. Hence,

$$
\begin{aligned}
\limsup _{n \rightarrow \infty} \frac{d_{D}\left(y_{n}+h_{n} z_{t}\right)-d_{D}\left(y_{n}\right)}{h_{n}} & =\limsup _{n \rightarrow \infty} \frac{d_{D}\left(y_{n}+h_{n} z_{n}\right)-d_{D}\left(y_{n}\right)}{h_{n}} \\
& \leq \limsup _{n \rightarrow \infty} \frac{\left\|\bar{x}_{n}-y_{n}\right\|+d_{D}\left(\bar{x}_{n}+h_{n} z_{n}\right)-d\left(y_{n}\right)}{h_{n}} \\
& =0 .
\end{aligned}
$$

Thus, $z_{t} \in \partial d_{D}(y)^{-}$; hence, $\sigma_{\varphi}(y ; \bar{x}) \leq 0$ as required.

Situation (ii) is similar. The set $D$, being convex in a reflexive space $E$, is proximinal (with $U=E$ ); however, it is not clear whether the Liminf property (from Example 6.3) of $\pi_{D}$ is satisfied. Nevertheless, choose an arbitrary $\varepsilon>0$; take $\eta=\varepsilon / 2, x \in D, y \in B(x, \eta)$, and $t \in J$. There is $\bar{x} \in \pi_{D}(y)$. Take $z_{t} \in$ $C_{D}(\bar{x}) \cap \varphi(t, \bar{x})=T_{D}(\bar{x}) \cap \varphi(t, \bar{x})$. There exist sequences $h_{n} \rightarrow 0^{+}$and $z_{n} \rightarrow z_{t}$ such that $\bar{x}+h_{n} z_{n} \in D$. Therefore,

$$
d_{D}\left(y+h_{n} z_{n}\right) \leq\|\bar{x}-y\|+d_{D}\left(\bar{x}+h_{n} z_{n}\right)=\alpha:=d_{D}(y) .
$$

Thus, $y+h_{n} z_{n} \in D_{\alpha}:=\left\{z \in E \mid d_{D}(z) \leq \alpha\right\}$. In other words, $z_{t} \in T_{D_{\alpha}}(y)=C_{D_{\alpha}}(y)$ $=\partial d_{D}(y)^{-}$.

Theorem 6.6. Suppose that condition (6.11) is satisfied and

(i) either $D$ is strictly regular, or

(ii) $D$ is regular and the semigroup $U$ is nonexpansive. ( $U$ is nonexpansive if $\omega=0$.)

Then, for any $x_{0} \in D$, the set $S\left(x_{0}, t_{0}\right)$ is an $R_{\delta}$-set provided condition (2.3) is satisfied or $U$ is compact.

The proof of Theorem 6.6 is technically involved and long. It resembles the above sketched arguments with important modifications: we show that $\varphi$ may be appropriately extended to a map $\varphi_{n}$ defined on a neighborhood $D_{n}:=\{x \in E \mid$ $\left.d_{D}(x, D) \leq \eta_{n}\right\}$ (where $\eta_{n} \rightarrow 0$ as $n \rightarrow \infty$ ), possessing a locally Lipschitz selection $f_{n}: J \times D_{n} \rightarrow E$ such that $d_{D}^{\circ}\left(x ; f_{n}(t, x)\right) \leq 0$ for $(t, x) \in J_{k} \times D_{n}$ for any $n, k \geq 1$ (where $J_{k}$ has the same meaning as in the proof of Theorem 4.4). Finally, we show that $S^{k}\left(x_{0}, t_{0}\right)$ is the intersection of the decreasing family of the contractible sets $S_{n}^{k}\left(x_{0}, t_{0}\right)$ of all mild solution on $J_{k}$ of (1.1) with $D$ replaced by $D_{n}$ and $\varphi$ by $\varphi_{n}$. The additional property of $U$ undertaken in (ii) helps establish the invariance of $D_{n}$. In case (i), the strict regularity allows to do without it. 
Remark 6.7. Suppose that $\varphi: J \times U \multimap E$, where $U$ is a neighborhood of a strictly regular $D$, satisfies assumptions enlisted at the beginning of the paper. If condition (2.3) holds (with $U$ replacing $D$ ) or the semigroup is compact, then $S\left(x_{0}, t_{0}\right)$ is an $R_{\delta}$-set for all $\left(x_{0}, t_{0}\right) \in D \times J$ provided the uniform tangency condition (6.11) is replaced by the exterior tangency condition

$$
\forall y \in \Omega \backslash D, \forall t \in J, \quad F(t, y) \cap \partial d_{D}(y)^{-} \neq \varnothing
$$

It seems that condition (6.14) is better designed to study the viability issues than (6.11) for it is simpler but requires that $\varphi$ is defined outside $D$.

\section{The periodic problem}

Let $0, T \in J$ where $T>0$. We now apply the preceding results in order to study the periodic problem considered as the two-point boundary value problem

$$
u^{\prime}(t) \in A u(t)+\varphi(t, u(t)), \quad x(0)=x(T)
$$

(by a solution of (7.1), we mean a mild solution of $u^{\prime}(t) \in A u(t)+\varphi(t, u(t))$ such that $u(0)=u(T)$ and $u: J \rightarrow D)$. In that, the method of the translation operator plays an important role, see, for example, [14, 37, 48]. The extension of this method to the case of differential inclusions (or equations) in infinite dimensional spaces is limited by the fact that the translation operator possesses the sufficient compactness properties only in exclusive cases (see [47]).

In view of Theorem 2.1, if (2.1) and (2.2) hold or the semigroup $U$ is compact, then we associate with (7.1) the set-valued Poincaré translation operator along trajectories $P=P_{T}: D \multimap D$ given by $P:=e_{T} \circ S$, where $S: D \multimap C(J, D)$, $S(x)=S(x, 0)$, is the solution operator and $e_{T}: C(J, D) \rightarrow D$ is the evaluation mapping $e_{T}(u):=u(T)$. Observe that $P$ maps bounded sets onto bounded ones. Clearly, the existence of periodic solutions is equivalent to the existence of fixed points of $P$.

Suppose that there is $\theta \in \mathbb{R}$ such that, for any $t>0$,

$$
\|U(t)\|^{\beta}:=\beta(U(t) B(0,1)) \leq \exp (\theta t) .
$$

Since $\|U(t)\|^{\beta} \leq\|U(t)\|$, the number $\theta$ always exists and $\theta \leq \omega$. Note that, for a bounded $\Omega \subset E, \beta(U(t) \Omega) \leq\|U(t)\|^{\beta} \beta(\Omega)$.

Theorem 7.1. Assume that $P$ is well defined, and let $\Omega \subset E$ be bounded.

(i) If the semigroup $U$ is compact, then $P(\Omega)$ is precompact. Thus, $P$ is a compact operator. 
(ii) Suppose that (2.3) holds. Then,

$$
\beta(P(\Omega)) \leq \exp \left(\theta T+\|k\|_{L^{1}}\right) \beta(\Omega) .
$$

This result improves some results obtained in [10] (see also [46] for analytic semigroups) and gives means to establish conditions necessary for $P$ to be a $\nu$ set contraction. If $\theta T+\|k\|_{L^{1}}<0$, then $P$ (if defined) is a $\nu$-set contraction (with respect to $\beta)$ with $v:=\exp \left(\theta T+\|k\|_{L^{1}}\right)$. Moreover, in the situation of Theorems $4.4,5.4$, and 6.6 or Remark 6.7, $P$ is a decomposable map.

Let $X$ be a metric space. A set-valued map $\Phi: X \multimap X$ is decomposable (see $[37,49])$ if there are a metric ANR $M$, an upper-semicontinuous set-valued map $\psi: X \multimap M$ such that, for every $x \in X$, the set $\psi(x) \subset M$ is $R_{\delta}$ and a continuous (single-valued map) $f: M \rightarrow X$ such that $\Phi=f \circ \psi$.

If, for any $x \in D, S(x)$ is an $R_{\delta}$-set, then $P$ is a decomposable map. Additionally, $P$ is homotopic (through a decomposable homotopy) to the identity $\operatorname{id}_{D}: D \rightarrow D$; the homotopy is provided by the composition $D \times[0,1] \ni(x, \lambda) \mapsto$ $\Psi(x, \lambda)=e_{\lambda T} \circ S(x)$ where $e_{\lambda T}$ is the evaluation $e_{\lambda T}(u):=u(\lambda T)$ for $u \in C(J, D)$; then, $\Psi(x, 0)=x$ and $\Psi(x, 1)=P$.

The class of decomposable maps falls into a much broader class of admissible maps (see [36]) particularly well-designed for the fixed-point problems. Roughly speaking, an upper semicontinuous map $\Phi: X \multimap X$ is admissible if it admits a set-valued selection being a finite composition of acyclic maps (a map is acyclic if it is upper semicontinuous with nonempty compact acyclic values). Clearly, a decomposable map $\Phi=f \circ \psi$, where $\psi$ is as above, is admissible.

We have the following fixed-point result.

Proposition 7.2 [36, Chapter V]. If $X$ is an ANR and $\Phi: X \multimap X$ is a decomposable map, then $\Phi$ has a fixed point provided

(i) $X$ is compact and $\chi(X) \neq 0$ (where $\chi(X)$ stands for the Euler characteristic of $X$ defined in terms of the rational Čech homology with compact supports. For any compact ANR $X, \chi(X)$ is a well-defined integer. In particular, if $X$ is a compact absolute retract, then $\chi(X)=1)$, or

(ii) $X$ is acyclic (e.g., contractible) and $\Phi$ is compact (here, it means that $\overline{\Phi(X)}$ is compact).

Observe that, in Theorems 4.4, 5.4, 6.6, and Remark 6.7, assumptions concerning $D$ always imply that $D$ is a regular set. In [24], it was shown that strictly regular sets are neighborhood retracts (we construct a neighborhood retraction $r: U \rightarrow D$, where $U$ is a neighborhood of $D$ via some variational arguments); so, they are compact regular sets. If $D$ is epi-Lipschitz, then, by the very definition, each point in $D$ has a neighborhood which is an ANR; hence, by the Hanner theorem (see, e.g., [15, Theorem 5.1]), $D$ is an ANR. In particular, $\chi(D)$ is well defined whenever $D$ is compact. Proposition 7.2 applies and we get the following results. 
Theorem 7.3. Suppose that $D \subset E$ is compact and that one of the following conditions hold:

(i) $D$ is regular (in particular, epi-Lipschitz), $\chi(D) \neq 0$, and (3.4) or (6.14) is satisfied (recall that if $D$ is compact, then (3.4) implies (6.11); if $F$ is single valued, then (3.4) may be replaced by (2.1));

(ii) $D$ is convex and (2.1) holds.

Then problem (7.1) admits a solution.

In the case $A \equiv 0$, the above theorem gives a positive answer to the open problem 13.1 in [31].

In order to dispense with the compactness of $D$, we need to impose compactness of $U$ (observe that given a closed and bounded set $D$ and a compact map $f: J \times D \rightarrow E$, the translation operator along trajectories associated with the equation $x^{\prime}=f(t, x)$ is easily seen to be only a 1-set-contraction, and, therefore, Proposition 7.2 does not apply; Deimling has given an example of such a map without any periodic solutions-see [27] and Remark 6.7 below).

Theorem 7.4. Suppose that $U$ is compact and $D$ is bounded and acyclic. If

(i) D is strictly regular and (6.11) or (6.14) is satisfied, or;

(ii) $D$ is convex (i.e., automatically contractible) and (2.1) holds, or;

(iii) D is epi-Lipschitz and (3.4) holds,

then problem (7.1) admits a solution.

The compactness assumption on $\mathcal{U}$ may still be relaxed.

Theorem 7.5. If $D$ is convex bounded, (2.1) and (2.2) hold, and $\theta T+\|k\|_{L^{1}}<0$, then (7.1) has a solution.

Proof. Theorems 4.4 and 7.1 imply that $P$ is a decomposable $\nu$-set contraction with $v<1$. In order to complete the proof, we will evoke the following result (see, e.g., $[9,57]$ ).

If $D$ is convex, closed and bounded, $\Phi: D \multimap D$ is a decomposable v-set contraction with respect to some regular, monotone and nonsingular measure of noncompactness $\gamma$ with $\nu<1$, then $\Phi$ has a fixed point.

Remark 7.6. In fact, we can do much better. Following Nussbaum [52], we suppose that a bounded and closed set $D \in \mathscr{F}$, that is, assume that $D=\bigcup_{i \in I} D_{i}$ where $\left\{D_{i}\right\}_{i \in I}$ is a locally finite family of closed convex subsets of $E$. Combining methods of [52] with those from [36] and given a decomposable $v$-set contraction (with $v<1$ ) $\Phi: D \multimap D$, we are in a position to define an integer valued invariant $i(\Phi, D)$ such that if $i(\Phi, D) \neq 0$, then $\Phi$ possesses a fixed point. Then, we show (see, e.g., [52, Section E, Theorem 4] for a hint) that if $D$ is acyclic, then $i(\Phi, D)=1$. Having this, we easily see that we may replace the convex bounded $D$

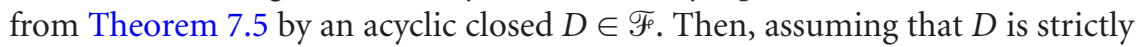
regular (It is, for instance, not difficult to get conditions implying that a finite 
union of closed convex sets is strictly regular.) and condition (6.11) is satisfied, we obtain a generalization of Theorem 7.5.

In Theorem 7.5, $\theta<0$ (this holds, e.g., if $\omega<0$ ). In the next result, we may also consider the case $\omega=0$.

THeOREM 7.7. Let the assumptions in front of Theorem 7.5 be fulfilled. Then, the periodic problem (7.1) has a mild solution provided $0 \in D, \omega \leq 0, \varphi$ is compact (i.e., $\overline{\varphi([0, T] \times D)}$ is compact) and 1 is in the resolvent set $\rho(U(T))$ of $U(T)$.

Proof. For $\varepsilon>0$, we consider the equation $y^{\prime} \in A y-\epsilon y+\varphi(t, y)$. Then, the $C_{0}-$ semigroup $U_{\varepsilon}:=\left\{U_{\varepsilon}(t)\right\}_{t \geq 0}$, where $U_{\varepsilon}(t)=e^{-\varepsilon t} U(t)$, generated by $A-\varepsilon I$, satisfies $U_{\varepsilon}(t) D \subset D$ for any $t \geq 0$ since $0 \in D$. By Theorem 7.5, we thus get the existence of a periodic solution $u_{\epsilon}$ to the perturbed equation. Since the resolvent set $\varrho(U(T))$ is open, we have the invertibility of $I-U_{\epsilon}(T)$ for $\epsilon>0$ sufficiently small. Thus, the following representation holds:

$$
u_{\varepsilon}(t)=U_{\varepsilon}(t)\left(I-U_{\varepsilon}(T)\right)^{-1} \int_{0}^{T} U_{\varepsilon}(T-s) w_{\varepsilon}(s) d s+\int_{0}^{t} U_{\varepsilon}(t-s) w_{\varepsilon}(s) d s
$$

for each $t \in J$ with some $w_{\varepsilon} \in N_{\varphi}\left(u_{\epsilon}\right)$.

Let $\varepsilon_{n} \succ 0$. Since $\varphi$ is compact and using (7.4), the Arzela-Ascoli theorem shows that $\left\{u_{\varepsilon_{n}}: n \geq 1\right\}$ is relatively compact in $C(J, E)$. Hence, without loss of generality, $u_{\varepsilon_{n}} \rightarrow u \in C(J, E)$. Clearly, $u$ is a mild solution of (7.1).

Remark 7.8. (1) Theorems 7.1, 7.3, 7.4, 7.5, and 7.7 contain the results on periodic solutions given in [11] as particular cases. Specialized to single-valued maps, our Theorems 7.5 and 7.7 improve [55, Theorems 3 and 4], where, as additional conditions, " $D$ has nonempty interior" and "the metric projection on $D$ exists," respectively, were considered.

(2) We may formulate the following periodic existence theorem of the Browder type. Suppose now that $E$ is a separable Hilbert space with the inner product $\langle\cdot, \cdot\rangle$. If $(2.3)$ holds and there is $r>0$ such that

$$
\forall t \in J, x \in E,\|x\|=r \exists z \in \varphi(t, x), \quad\langle z, x\rangle \leq 0,
$$

then the periodic problem (7.1) has a mild solution provided $\omega T+\|k\|_{L^{1}}<0$.

Let $D:=B(0, r)$. Then, (7.5) exactly means that $\varphi(t, x) \cap T_{D}(x) \neq \varnothing$ on $J \times D$. Since $\omega<0, \mathcal{U}$ is nonexpansive and $U(t) D \subset D$ for each $t \geq 0$. Thus, Theorem 7.5(ii) shows the existence of the periodic solution.

(3) We recall [28, Example 24.12]; let $f: \mathbb{R} \times D \rightarrow E$ and $g: \mathbb{R} \times D \rightarrow E$ be continuous and $T$-periodic in the first variable such that $\alpha(f(\{t\} \times \Omega)) \leq$ $k_{1}(t) \alpha(\Omega)$ for all bounded $\Omega \subset D$ and

$$
\forall x, y \in D, \quad(g(t, x)-g(t, y), x-y)_{-} \leq k_{2}(t)\|x-y\|^{2},
$$


where $(\cdot, \cdot)_{-}$denotes the semi-inner product $(\cdot, \cdot)_{-}: E \times E \rightarrow \mathbb{R}$ given by

$$
(x, y)_{-}:=\|y\| \lim _{t \rightarrow 0^{+}} \frac{\|y\|-\|y-t x\|}{t}
$$

$f_{0}:=f+g$ is uniformly continuous and bounded and satisfies (2.1); the functions $k_{1}, k_{2} \in L^{1}(J)$ and $k:=\int_{0}^{T}\left(k_{1}(s)+k_{2}(s)\right) d s<0$. In [28], existence of periodic orbits is shown in case $D$ as having nonempty interior. We may dispense with this assumption: as in the proof of Theorem 4.4, we also show that in this situation where the set of continuously differentiable solutions is an $R_{\delta}$-set. The translation operator along the trajectories $P: D \multimap D$ satisfies $\beta(P(\Omega)) \leq e^{k} \beta(\Omega)$ for bounded $\Omega \subset D$ (see [28]). Hence, we may again apply the fixed-point result stated in the proof of Theorem 7.5 to show the existence of a fixed point of $P$, that is, a periodic solution.

Assumptions in Theorems 7.4, 7.5, and 7.7 implicitly require that the linear part $A$ is nonzero (except for Theorem 7.3 where $D$ is compact). We now deal with a noncompact domain not excluding the case $A \equiv 0$, but our discussion will be specialized to strongly continuous nonlinearities.

A set-valued map $\Phi: D \multimap E$ is strongly upper semicontinuous if, for every weakly convergent sequence $x_{n} \rightarrow x_{0}$ in $D$, a sequence $y_{n} \in \Phi\left(x_{n}\right)$ has a subsequence $y_{n_{k}} \rightarrow y_{0} \in \Phi\left(x_{0}\right)$.

The notion of a strongly upper semicontinuous map was first introduced in [34] under the name of completely continuous maps (however not in connection with differential equations or inclusions). Obviously, strong upper semicontinuity implies upper semicontinuity.

Example 7.9. If $E^{\prime}$ is a Banach space, $j: E \rightarrow E^{\prime}$ is a compact bounded linear map, and $\psi: E^{\prime} \multimap E$ is upper semicontinuous, then $\Phi:=\psi \circ j \mid D: D \multimap E$ is strongly upper semicontinuous. For if $x_{n} \rightarrow x_{0} \in D$, then $j\left(x_{n}\right) \rightarrow j\left(x_{0}\right)$ and the strong upper semicontinuity follows from the upper semicontinuity of $\psi$. In particular, if $E \hookrightarrow \hookrightarrow E^{\prime}$ (i.e., $E$ is compactly embedded into $E^{\prime}$ ) and $\psi: E^{\prime} \multimap E$ is upper semicontinuous, then $\Phi=\psi \mid D$ is strongly upper semicontinuous.

Our interest in strongly upper semicontinuous maps is motivated by the following fixed-point result.

Proposition 7.10. Let $D \subset E$ be a weakly compact convex, and let $\Phi: D \multimap D$ be a decomposable map. Assume there is a linear, bounded operator $U: E \rightarrow E,\|U\| \leq 1$ and a strongly upper semicontinuous map $\psi: D \multimap$ E such that

$$
\forall x \in D \quad \Phi(x)=U x+\psi(x)
$$

Then, $\Phi$ has a fixed point. 
The proof follows arguments given in [9, Corollary 11]. Note that in Proposition 7.10, we cannot replace the strong upper semicontinuity of $\psi$ by the upper semicontinuity and compactness of $\psi$. To see this, let $E:=\ell^{2}, D:=$ $\overline{B(0,1)} \subset \ell^{2}, U: E \rightarrow E$ be given by $U(x):=\left(0, x_{1}, x_{2}, \ldots\right)$ and $\psi(x):=(1-\|x\|$, $0, \ldots)$. Then, $\Phi:=T+\varphi: D \rightarrow D$, but it has no fixed points.

Theorem 7.11. Assume that $U$ is uniformly continuous and $\|U(T)\| \leq 1$. If $D$ is convex and weakly compact, $\varphi$ satisfies (2.1) and (2.2) and that, for all $t \in[0, T]$, $\varphi(t, \cdot)$ is strongly upper semicontinuous, then problem (7.1) has a strong solution.

As stated above, the case $A=0$ is not excluded. Thus, in view of the remark following Theorem 7.3, compactness of the nonlinearity is not sufficient for the existence of periodic solutions. This is why the stronger assumption is considered here.

Proof. We apply Proposition 7.10; to this end, introduce the map $\psi: D \multimap E$,

$$
\varphi(x):=\left\{\int_{0}^{T} U(T-s) w(s) d s \mid w(\cdot) \in F(\cdot, u(\cdot)), u \in S(x)\right\},
$$

for $x \in D$, and let $U:=U(T)$. We easily see that

$$
P(x)=U x+\psi(x) \quad \text { on } D
$$

Our assumptions concerning $\varphi$ imply that all the assumptions of Theorem 4.4 are fulfilled; hence, $P$ (and $\psi$ ) is decomposable. In view of representation (7.10), we conclude the proof of the existence of a mild solution of (7.1) by showing that $\psi$ is strongly upper semicontinuous. In order to get a strong solution, we use the uniform continuity of $u$.

Remark 7.12. If $\varphi$ is single-valued, then we do not need the uniform continuity of $U$. In this case, we assume that $E$ is reflexive and $D$ is convex bounded (thus weakly compact), and, in order to get the existence of a mild solution, the weak equicontinuity of $\{U(\cdot) x\}_{x \in D}$ is shown and employed. In particular, if $\varphi$ is single-valued and (jointly) strongly continuous and $A \equiv 0$, then the existence of a classical solution of the problem

$$
u^{\prime}=f(t, u), \quad u(0)=u(T)
$$

is easily obtained (reflexivity is not relevant for it was necessary only to establish the weak equicontinuity of $\left.\{U(\cdot)\}_{x \in D}\right)$.

\section{Equilibria}

Let $F: D \multimap E$. A stationary solution of the autonomous inclusion

$$
u^{\prime}(t) \in A u(t)+\Phi(u(t))
$$


that is, a point $u_{0} \in D(A) \cap D$ satisfying $0 \in A u_{0}+F\left(u_{0}\right)$, is called an equilibrium of (8.1). The existence of equilibria (for $A \equiv 0$ ) has been carefully studied in [24] by different methods and in [13] in a finite-dimensional setting (see also [8] and others). Here, we assume that $\Phi$ is upper semicontinuous and have nonempty, convex and compact values. Moreover, we assume that $\Phi$ is subject to one of the following tangency conditions:

$$
\begin{array}{ll}
\forall x \in D, & \Phi(x) \cap T_{D}(x) \neq \varnothing ; \\
\forall x \in D, & \Phi(x) \cap C_{D}(x) \neq \varnothing ;
\end{array}
$$

that is, "autonomous" analogs of conditions (2.1) and (3.4), respectively.

Theorem 8.1. Suppose that D is compact. Then, (8.1) has an equilibrium provided

(i) D is regular (in particular, epi-Lipschitz), $\chi(D) \neq 0$, and (8.3) is satisfied; or

(ii) $D$ is convex and (8.2) holds.

Proof. Fix $T>0$. By Theorem 7.3, for each $n \geq 1$, there is a $2^{-n} T$-periodic mild solution $u_{n}$ of (8.1). The compactness of $D$ shows that (passing to a subsequence) $u_{n} \rightarrow u \in C([0, T], E)$ and $u$ is a mild solution of (8.1). Clearly, $u(t) \equiv u_{0} \in D$. Thus,

$$
u_{0}=U(t) u_{0}+\int_{0}^{t} U(t-s) w(s) d s, \quad t \in[0, T]
$$

where $w(s) \in \Phi\left(u_{0}\right)$ on $[0, T]$. The function $v(t)=\int_{0}^{t} w(s) d s$ is differentiable a.e; suppose that for $z \in(0, T), v^{\prime}(z)=w(z)=: y_{0} \in \Phi\left(u_{0}\right)$ exists. By $(8.4)$, for $h>0$, $u_{0}=U(h) u_{0}+\int_{z}^{z+h} U(z+h-s) w(s) d s$. Hence,

$$
\frac{U(h) \mathcal{u}_{0}-u_{0}}{h}=\frac{1}{h} \int_{z}^{z+h}(w(s)-U(z+h-s) w(s)) d s-\frac{v(z+h)-v(z)}{h} .
$$

The first term in the right-hand side tends to 0 as $h \rightarrow 0^{+}$, and the second one converges to $-y_{0}$. Hence, $u_{0} \in D(A)$ and $A u_{0}=-y_{0}$.

In a similar manner, we get the next results.

THEOREM 8.2. Let $D$ be bounded and acyclic. If the semigroup is compact and one of the following conditions holds

(i) D is strictly regular, $\Phi$ is defined on a neighborhood $U$ of $D$, and

$$
\forall y \in U \quad \Phi(y) \cap \partial d_{D}(y)^{-} \neq \varnothing ;
$$

(ii) D is epi-Lipschitz and (8.3) holds; or

(iii) $D$ is convex and (8.2) holds,

then (8.1) has an equilibrium. 
Theorem 8.3. Let D be convex bounded and (8.2) hold. Then, (8.1) has an equilibrium provided one of the following conditions is satisfied:

(i) $\Phi$ is a $v$-set contraction, $\theta+\nu<0$;

(ii) $0 \in D, \omega \leq 0$, $\Phi$ is compact and there is $t_{0}>0$ such that $1 \in \varrho(U(t))$ for each $0<t<t_{0}$;

(iii) $\Phi$ is strongly upper semicontinuous, $D$ is weakly compact, and $U$ is uniformly continuous and nonexpansive;

(iv) $\Phi$ is single-valued strongly continuous, $U$ is nonexpansive, and $E$ is reflexive.

For the proof, observe that if $\varphi$ (independent of $t$ ) is a $\nu$-set contraction, then (2.2) holds true (on $[0, T])$ with $k(\cdot) \equiv v$. Hence, $\|k\|_{L^{1}([0, T])}=v T$, and it follows that $\theta T+\nu T<0$ for each $T>0$. Then, we can proceed exactly as in the proof of Theorem 8.1. That $\left(u_{n}\right)$ has a convergent subsequence follows since $\left\{u_{n}(0)\right\}$, as the fixed point set of an appropriate Poincaré operator, is compact.

Observe that if $D$ is strictly regular, then we may easily state an "autonomous" version of (6.11) and obtain a different result asserting the existence of equilibria. At the same time in Theorems 8.1(i) or 8.2(ii), condition (8.3) may be replaced by (8.2) if $\Phi$ is single-valued.

Remark 8.4. (1) Let $f: \ell^{2} \rightarrow \ell^{2}$ be defined by $f(x)=y$ where $y_{n}:=\alpha_{n} x_{n}+$ $\beta_{n}(\|x\|-1)^{2}$ for $n \geq 1$, where $\alpha_{n} \rightarrow 0, \alpha_{n}<0,\left(\beta_{n}\right) \in \ell^{2}$, and $\left(\beta_{n} / \alpha_{n}\right) \notin \ell^{2}$. Then, $f$ is compact and satisfies $\langle f(x), x\rangle<0$ for each $x \in \ell^{2}$ with $\|x\|=1$; hence, $f(x) \in T_{D}(x)$ on the unit ball $D$. However, the problem $u^{\prime}(t)=f(u(t))$ has no solution of any period (see [27]). In view of Remark 7.12, it is clear that $f$ is not strongly continuous. Indeed, let $e_{m}:=\left(\delta_{n m}\right)$. Then, $e_{m} \stackrel{\ell^{2}}{\rightarrow} 0, f\left(e_{m}\right)=$ $\left(0, \ldots, 0, \alpha_{m}, 0, \ldots\right)$ and thus, $f\left(e_{m}\right) \stackrel{\ell^{2}}{\rightarrow} 0$ as $m \rightarrow \infty$. But $f(0)=\left(\beta_{m}\right) \neq 0$. However, problem $0=f(x)$ has a solution in case $\alpha_{n} \rightarrow \omega<0$. This follows from Theorem 8.3(ii).

(2) In [28, page 211], it is conjectured that if $D \subset E$ is closed, bounded and convex, $f, g: D \rightarrow E$ are continuous and bounded, $\alpha(f(\Omega)) \leq \nu_{1} \alpha(\Omega)$ for all $\Omega \subset$ $D$ and

$$
(g(x)-g(y), x-y)_{-} \leq v_{2}\|x-y\|^{2} \quad \text { for each } x, y \in D
$$

and $f+g$ is weakly inward (i.e., $\Phi:=f+g-\mathrm{id}_{E}$ satisfies $(8.2)$ ), then $f+g$ has a fixed point provided that $\nu_{1}+\nu_{2}<1$.

We may prove this conjecture under the additional assumption that $f+g$ is uniformly continuous improving previous results (see [28]). Indeed, let $g_{0}:=$ $g-\mathrm{id}_{E}$; the properties of the semi-inner product $(\cdot, \cdot)_{-}$imply that $g_{0}$ satisfies the estimate (8.7) with $\nu_{2}-1$ replacing $\nu_{2}$. For each $T>0, k:=T\left(\nu_{1}+\left(\nu_{2}-1\right)\right)<0$ and hence, by the result given in Remark 7.8(3), the existence of a T-periodic solution of $u^{\prime}(t)=F(u(t))$ follows. Arguing as in the proof of Theorem 8.3, we get a constant solution $u(t) \equiv u$, which means $0=\Phi(u)$, that is, $u=f(u)+g(u)$. 


\section{Final remarks}

We observe that, in most of the above results, we can do without the separability of $E$ assuming that $\varphi$ is upper semicontinuous or even almost upper semicontinuous in the sense of Deimling (see [31]) although the proofs are a bit more involved. The important changes have to be done in Theorem 7.1, if $E$ is an arbitrary (resp., weakly compactly generated) Banach space, then the Poincaré operator is a $\nu$-set contraction with respect to the measure of noncompactness $\widetilde{\beta}_{0}$ on $E$ given by

$$
\widetilde{\beta}_{0}(\Omega):=\sup \{\beta(C) \mid C \subset \Omega \text { countable }\}
$$

for a bounded $\Omega \subset E\left(\widetilde{\beta}_{0}\right.$ is regular, monotone and nonsingular (see [1, Section 1.4]) with $v=\theta T+4\|k\|_{L^{1}}$ (resp., $v=\theta T+2\|k\|_{L^{1}}$ ). Consequently, changing assumptions in Theorems 7.5 and 8.3(i), we obtain other criteria for the existence of periodic orbits or equilibria.

The detailed proofs of the main results of this paper will appear in the forthcoming paper [12].

\section{Acknowledgments}

This paper is an extended version of the talk given at the International Conference on Fixed-Point Theory and Its Applications, Haifa 2001. The author was supported in part by the Faculty of Mathematics and Computer Sciences Research Statute Fund and the KBN Grant no. 2 P03A 02416.

\section{References}

[1] R. R. Akhmerov, M. I. Kamenskiĭ, A. S. Potapov, A. E. Rodkina, and B. N. Sadovskiü, Measures of Noncompactness and Condensing Operators, Operator Theory: Advances and Applications, vol. 55, Birkhäuser Verlag, Basel, 1992.

[2] J. Andres, G. Gabor, and L. Górniewicz, Topological structure of solution sets to multivalued asymptotic problems, Z. Anal. Anwendungen 19 (2000), no. 1, 35-60.

[3] Acyclicity of solution sets to functional inclusions, Nonlinear Anal. Ser. A: Theory Methods 49 (2002), no. 5, 671-688.

[4] N. Aronszajn, Le correspondant topologique de l'unicité dans la théorie des équations différentielles, Ann. of Math. (2) 43 (1942), 730-738 (French).

[5] J.-P. Aubin, A survey of viability theory, SIAM J. Control Optim. 28 (1990), no. 4, 749-788.

[6] - Viability Theory, Systems \& Control: Foundations \& Applications, Birkhäuser Boston, Massachusetts, 1991.

[7] J.-P. Aubin and A. Cellina, Differential Inclusions, Grundlehren der Mathematischen Wissenschaften, vol. 264, Springer-Verlag, Berlin, 1984.

[8] J.-P. Aubin and H. Frankowska, Set-Valued Analysis, Systems \& Control: Foundations \& Applications, vol. 2, Birkhäuser Boston, Massachusetts, 1990.

[9] R. Bader, Fixed point theorems for compositions of set-valued maps with single-valued maps, Ann. Univ. Mariae Curie-Skłodowska Sect. A 51 (1997), no. 2, 29-41. 
[10] The periodic problem for semilinear differential inclusions in Banach spaces, Comment. Math. Univ. Carolin. 39 (1998), no. 4, 671-684.

[11] On the semilinear multi-valued flow under constraints and the periodic problem, Comment. Math. Univ. Carolin. 41 (2000), no. 4, 719-734.

[12] R. Bader and W. Kryszewski, On the solution sets of differential inclusions and the periodic problem in Banach spaces, to appear in Nonlinear Anal. Ser. A: Theory Methods.

[13] On the solution sets of constrained differential inclusions with applications, Set-Valued Anal. 9 (2001), no. 3, 289-313.

[14] H. Ben-El-Mechaiekh and W. Kryszewski, Equilibria of set-valued maps on nonconvex domains, Trans. Amer. Math. Soc. 349 (1997), no. 10, 4159-4179.

[15] C. Bessaga and A. Pełczyński, Selected Topics in Infinite-Dimensional Topology, Monografie Matematyczne, vol. 58, PWN-Polish Scientific Publishers, Warsaw, 1975.

[16] D. Bothe, Multivalued differential equations on graphs and applications, Ph.D. thesis, Universität Paderborn, 1992.

[17] _ Periodic solutions of nonlinear evolution problems, manuscript, 1997.

[18] Multivalued perturbations of m-accretive differential inclusions, Israel J. Math. 108 (1998), 109-138.

[19] O. Cârjă and I. I. Vrabie, Some new viability results for semilinear differential inclusions, NoDEA Nonlinear Differential Equations Appl. 4 (1997), no. 3, 401-424.

[20] M. Cichon and I. Kubiaczyk, Some remarks on the structure of the solution set for differential inclusions in Banach spaces, J. Math. Anal. Appl. 233 (1999), no. 2, 597-606.

[21] F. H. Clarke, Optimization and Nonsmooth Analysis, Canadian Mathematical Society Series of Monographs and Advanced Texts, John Wiley \& Sons, New York, 1983.

[22] G. Conti, V. Obukhovskilı, and P. Zecca, On the topological structure of the solution set for a semilinear functional-differential inclusion in a Banach space, Topology in Nonlinear Analysis (Warsaw, 1994), Banach Center Publ., vol. 35, Polish Acad. Sci., Warsaw, 1996, pp. 159-169.

[23] B. Cornet and M.-O. Czarnecki, Représentations lisses de sous-ensembles épilipschitziens de $\mathbf{R}^{n}$, C. R. Acad. Sci. Paris Sér. I Math. 325 (1997), no. 5, 475-480 (French).

[24] A. Ćwiszewski and W. Kryszewski, Equilibria of set-valued maps: a variational approach, Nonlinear Anal. Ser. A: Theory Methods 48 (2002), no. 5, 707-746.

[25] J. L. Davy, Properties of the solution set of a generalized differential equation, Bull. Austral. Math. Soc. 6 (1972), 379-398.

[26] F. S. De Blasi and J. Myjak, On the solutions sets for differential inclusions, Bull. Polish Acad. Sci. Math. 33 (1985), no. 1-2, 17-23.

[27] K. Deimling, Periodic solutions of differential equations in Banach spaces, Manuscripta Math. 24 (1978), no. 1, 31-44.

[28] - Nonlinear Functional Analysis, Springer-Verlag, Berlin, 1985.

[29] - On solution sets of multivalued differential equations, Appl. Anal. 30 (1988), no. 1-3, 129-135.

[30] - Extremal solutions of multivalued differential equations. II, Results Math. 15 (1989), no. 3-4, 197-201.

[31] _ Multivalued Differential Equations, de Gruyter Series in Nonlinear Analysis and Applications, vol. 1, Walter de Gruyter, Berlin, 1992.

[32] T. Donchev, Semicontinuous differential inclusions, Rend. Sem. Mat. Univ. Padova 101 (1999), 147-160. 
[33] R. Dragoni, J. W. Macki, P. Nistri, and P. Zecca, Solution Sets of Differential Equations in Abstract Spaces, Pitman Research Notes in Mathematics Series, vol. 342, Longman, Harlow, 1996.

[34] P. M. Fitzpatrick and W. V. Petryshyn, A degree theory, fixed point theorems, and mapping theorems for multivalued noncompact mappings, Trans. Amer. Math. Soc. 194 (1974), 1-25.

[35] G. Gabor, On the acyclicity of fixed point sets of multivalued maps, Topol. Methods Nonlinear Anal. 14 (1999), no. 2, 327-343.

[36] L. Górniewicz, Homological methods in fixed-point theory of multi-valued maps, Dissertationes Math. (Rozprawy Mat.) 129 (1976), 71.

[37] - Topological approach to differential inclusions, Topological Methods in Differential Equations and Inclusions (Montreal, PQ, 1994) (A. Granas and M. Frigon, eds.), NATO Adv. Sci. Inst. Ser. C Math. Phys. Sci., vol. 472, Kluwer Academic Publishers, Dordrecht, 1995, pp. 129-190.

[38] _ Topological structure of solution sets: current results, Arch. Math. (Brno) 36 (2000), no. suppl., 343-382.

[39] L. Górniewicz, P. Nistri, and V. Obukhovskii, Differential inclusions on proximate retracts of Hilbert spaces, Int. J. Non-linear Diff. Eq 3 (1997), 13-26.

[40] C. J. Himmelberg and F. S. Van Vleck, On the topological triviality of solution sets, Rocky Mountain J. Math. 10 (1980), no. 1, 247-252.

[41] A note on the solution sets of differential inclusions, Rocky Mountain J. Math. 12 (1982), no. 4, 621-625.

[42] S. C. Hu and N. S. Papageorgiou, On the topological regularity of the solution set of differential inclusions with constraints, J. Differential Equations 107 (1994), no. 2, 280-289.

[43] _ Handbook of Set-Valued Analysis, Vol. I, Kluwer Academic Publishers, 1999.

[44] Handbook of Set-Valued Analysis, Vol. II, Kluwer Academic Publishers, 2001.

[45] D. M. Hyman, On decreasing sequences of compact absolute retracts, Fund. Math. 64 (1969), 91-97.

[46] M. Kamenskii, V. Obukhovskii, and P. Zecca, Condensing Multivalued Maps and Semilinear Differential Inclusions in Banach Spaces, de Gruyter Series in Nonlinear Analysis and Applications, vol. 7, Walter de Gruyter, Berlin, 2001.

[47] M. I. Kamenskii and V. V. Obukhovskiĭ, On the operator of translation along the trajectories of semilinear controlled systems, Differ. Uravn. 32 (1996), no. 6, 747-754 (Russian).

[48] M. A. Krasnosel'skiı̌ and P. P. Zabrě̌ko, Geometrical Methods of Nonlinear Analysis, Grundlehren der Mathematischen Wissenschaften, vol. 263, Springer-Verlag, Berlin, 1984.

[49] W. Kryszewski, Homotopy invariants for set-valued maps. Homotopy approximation approach, Fixed Point Theory and Applications (Marseille, 1989) (M. A. Théra and J.-B. Baillon, eds.), Pitman Res. Notes Math. Ser., vol. 252, Longman Sci. Tech., Harlow, 1991, pp. 269-284.

[50] R. H. Martin Jr., Nonlinear Operators and Differential Equations in Banach Spaces, Pure and Applied Mathematics, John Wiley \& Sons, New York, 1976.

[51] D. Motreanu and N. Pavel, Tangency, Flow Invariance for Differential Equations, and Optimization Problems, Monographs and Textbooks in Pure and Applied Mathematics, vol. 219, Marcel Dekker, New York, 1999.

[52] R. D. Nussbaum, The fixed point index for local condensing maps, Ann. Mat. Pura Appl. (4) 89 (1971), 217-258. 
[53] A. Pazy, Semigroups of Linear Operators and Applications to Partial Differential Equations, Applied Mathematical Sciences, vol. 44, Springer-Verlag, New York, 1983.

[54] S. Plaskacz, On the solution sets for differential inclusions, Boll. Un. Mat. Ital. A (7) 6 (1992), no. 3, 387-394.

[55] J. Prüss, Periodic solutions of semilinear evolution equations, Nonlinear Anal. 3 (1979), no. 5, 601-612.

[56] R. T. Rockafellar, Clarke's tangent cones and the boundaries of closed sets in $\mathbf{R}^{n}$, Nonlinear Anal. 3 (1979), no. 1, 145-154.

[57] I. I. Vrabie, Compactness Methods for Nonlinear Evolutions, Pitman Monographs and Surveys in Pure and Applied Mathematics, vol. 32, Longman Scientific \& Technical, Harlow, 1987.

[58] K. Yosida, Functinal Analysis, Springer-Verlag, Berlin, 1966.

Wojciech Kryszewski: Faculty of Mathematics and Computer Science, Nicholas Copernicus University, ul. Chopina 12/18, 87-100 Torun, Poland; Faculty of Mathematics, University of Lodz, ul. Banacha 22, 90-238 Łódź, Poland

E-mail addresses: wkrysz@mat.uni.torun.pl; wkrysz@imul.math.uni.lodz.pl 


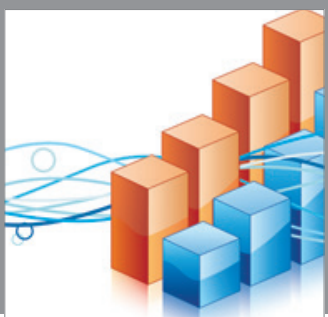

Advances in

Operations Research

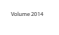

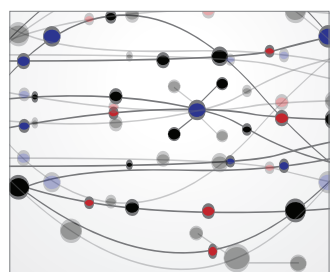

\section{The Scientific} World Journal
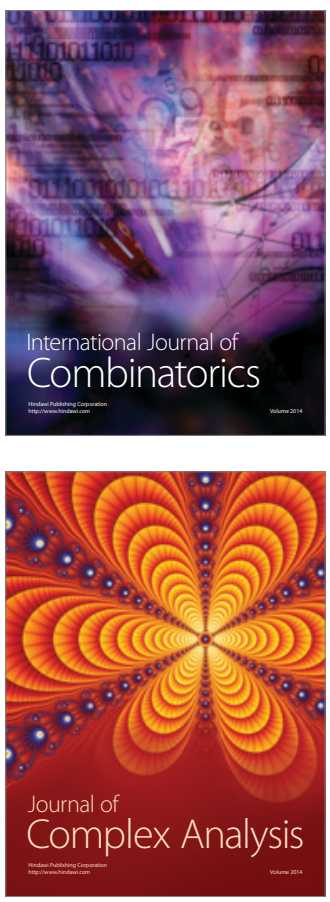

International Journal of

Mathematics and

Mathematical

Sciences
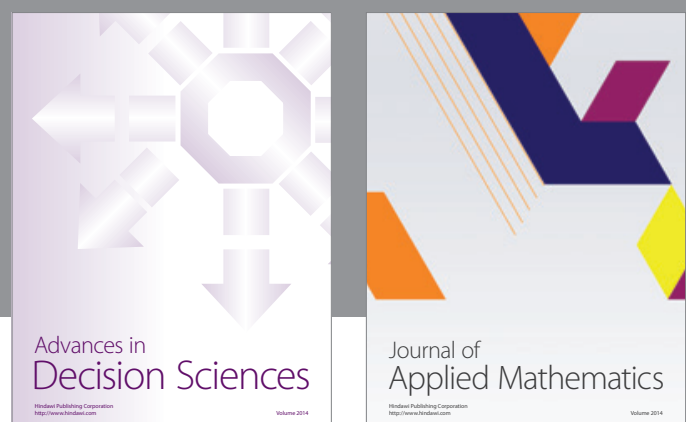

Journal of

Applied Mathematics
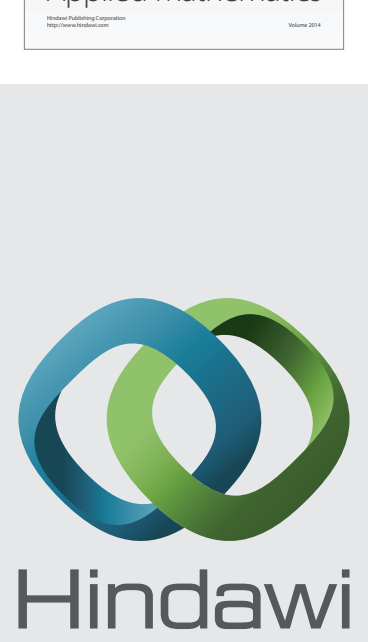

Submit your manuscripts at http://www.hindawi.com
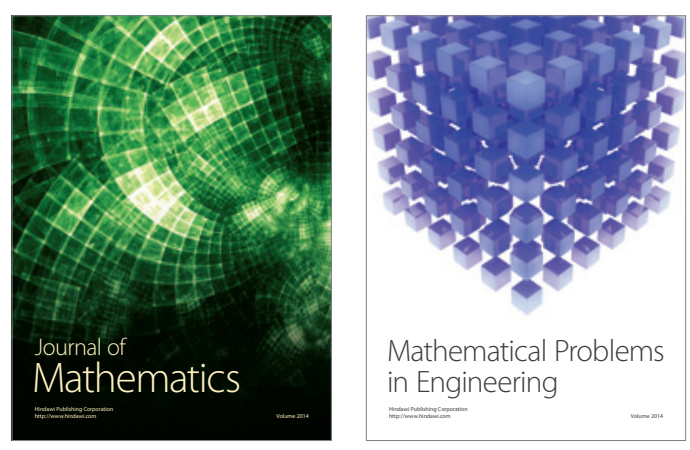

Mathematical Problems in Engineering
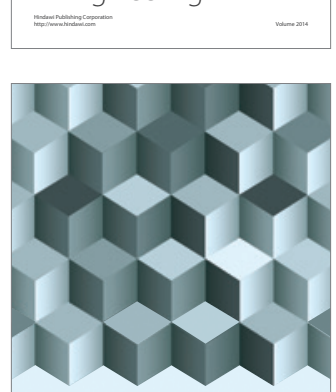

Journal of

Function Spaces
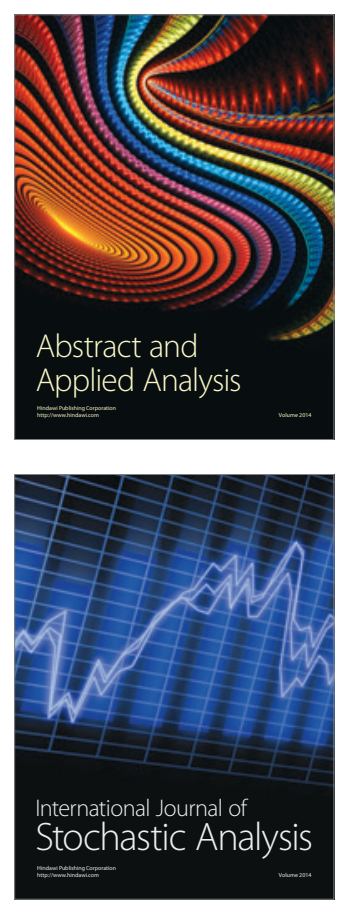

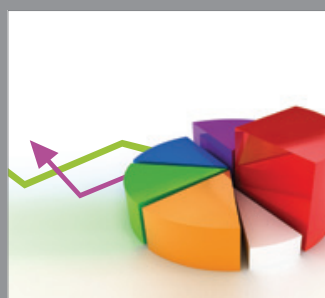

ournal of

Probability and Statistics

Promensencen
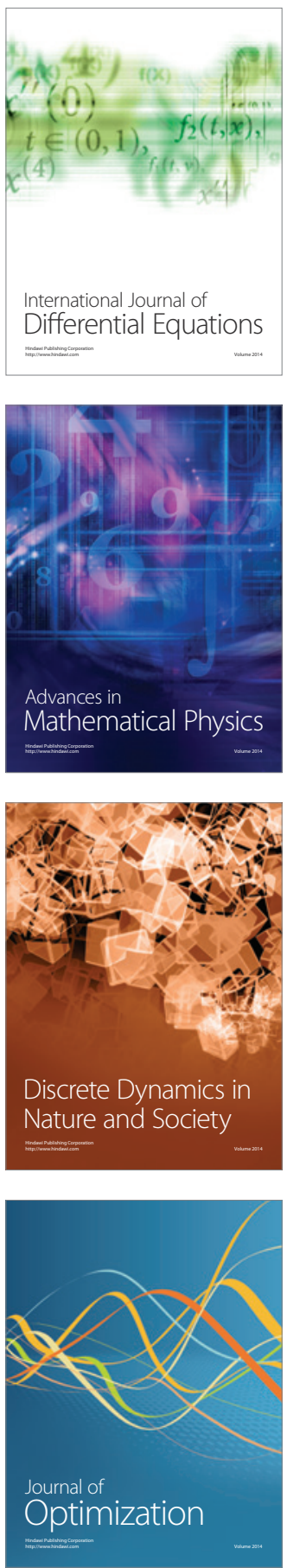